\title{
¿Es posible escapar del nacionalismo?
}

\author{
Modesto Gayo Cal
}

Universidad Diego Portales. Santiago de Chile

modesto.gayo@upd.cl

Recibido: 17-04-2008

Aceptado: 04-06-2009

\section{Resumen}

El nacionalismo ha sido considerado una ideología responsable de conflictos sociales, guerras y genocidios. Ha resultado habitual vincularla con una identidad colectiva que se transforma en acción a través del comportamiento de los individuos que la interiorizan. Habría, por tanto, nacionalistas y no nacionalistas. Sin embargo, en este artículo proponemos las siguientes ideas. En primer lugar, no es posible escapar del nacionalismo, porque se ha constituido en la ideología que promociona el principio fundamental sobre el que se instituyen los estados contemporáneos: la soberanía nacional. En segundo lugar, normalmente, el nacionalismo no es responsable de todos los males que se le achacan, sino que sirve para dar cobertura ideológica a problemas políticos que tienen otro origen. En tercer lugar, si estamos insatisfechos con el principio de nacionalidad, deberíamos ser capaces de sugerir alternativas sobre las cuales existen consensos iguales o superiores.

Palabras clave: nacionalismo, ideología, nación, identidad nacional, soberanía nacional.

\section{Abstract. Is it possible to escape nationalism?}

Nationalism has been treated as an ideology responsible for social conflicts, war and genocides. It has become a habit link it to a collective identity that turns into action through the behaviour of those people who have interiorised it. Therefore, there would be nationalists and non-nationalists. However, in this paper the following ideas are proposed: Firstly, we cannot escape nationalism because it has become the ideology that promotes the main principle over which contemporary states take shape: namely national sovereignty. Secondly, nationalism is frequently not responsible for all the troubles for which it is accused, but it has been used to give ideological backup to political problems with different origins. Thirdly, if we are not satisfied with the principle of nationality, we should be able to come up with widely accepted and viable alternatives.

Key words: nationalism, ideology, nation, national identity, national sovereignty. 


\author{
Sumario \\ I. Introducción VII. Las soluciones políticas \\ al nacionalismo \\ II. ¿Cómo se legitima la existencia \\ de los estados? \\ VIII. Notas sobre el caso español \\ III. ¿Qué es el nacionalismo? \\ IV. El relato sobre el surgimiento \\ del nacionalismo \\ IX. Finalmente, el nacionalismo \\ y la idenidad \\ X. Conclusiones \\ V. ¿Es tan peligroso el nacionalismo? \\ Referencias bibliográficas \\ VI. ¿Cuándo molesta el nacionalismo?
}

\title{
I. Introducción
}

En la modernidad política posterior a la Revolución Francesa, se ha dado una relación ambigua con el nacionalismo. Por un lado, desde el punto de vista de la reflexión sobre su origen, no ha habido acuerdo sobre la importancia de las condiciones propias de la modernidad (desarrollo económico, político, tecnológico) en el surgimiento del fenómeno aludido. Para unos, las bases sobre las que se constituye el nacionalismo serían premodernas o étnicas (Armstrong, 1982; Smith, 1986), mientras que, para otros, quizás la mayoría, estaríamos ante algo históricamente nuevo, de naturaleza mayormente política (Gellner, 1983; Brass, 1991; Hobsbawm, 1992, entre otros muchos $)^{1}$. Como resultado de este trabajo intelectual, tenemos aportaciones valiosas, pero, en conjunto, no ha habido una solución satisfactoria y de amplia aceptación, lo que ha hecho que sea más acertado hablar de debates que de descubrimientos ${ }^{2}$. Por otro lado, en lo que se refiere a sus consecuencias políticas, a lo largo del tiempo se ha producido un cambio importante. Si, en la primera parte del siglo XIX, fue bienvenido y abrazado con entusiasmo como fuente de liberación frente a la opresión extranjera (pensemos en los bien conocidos casos griego, italiano y alemán de la época), e incluso tras la Primera Guerra Mundial sirvió de principio para construir el nuevo mapa político europeo, la forma extraordinariamente belicista en la que se desenvolvieron los acontecimientos políticos durante el período de entreguerras y el descubrimiento del holocausto judío, asociado todo ello a regímenes que llevaban la idea de nación a su paroxismo, condujo a un descrédito acelerado del nacionalismo ${ }^{3}$. Es cierto que el principio político de nacionalidad fue rehabilitado parcialmente a través de los procesos de des-

1. También ha habido relecturas de la literatura sobre esta temática que han tratado de integrar elementos modernos y premodernos (Llobera, 1994).

2. Smith $(1998,2001)$ habla de paradigmas.

3. Un ejemplo de ello es la obra de E. Kedourie (1966), escrita durante la postguerra y fuertemente crítica con el nacionalismo. 
colonización que se produjeron durante las décadas posteriores a la Segunda Guerra Mundial, pero su aceptación nunca ha estado carente de importantes cuotas de escepticismo ${ }^{4}$.

En este escenario histórico de ambigüedad, han tenido un importante peso los que dicen repudiar a los nacionalismos como una manera de pensar retrógrada o poco adecuada en la época contemporánea, la cual estaría ligada a crímenes y a violencia políticos, como también a conflictos sociales en general $^{5}$. El patrón más común ha sido que los autores más críticos han sido aquéllos que han promovido la idea de la modernidad del nacionalismo, arriba indicada. Este entendimiento implica que estamos ante un fenómeno político, en primer lugar y antes de nada, e identitario, en segundo lugar y de forma residual. En este sentido, se sostendría, entre los argumentos más destacados, que estaríamos ante lo que podríamos llamar una producción de falsa conciencia en favor de la burguesía o de las clases dominantes en la sociedad (en la línea de la tradición marxista ${ }^{6}$, y Hobsbawm dentro de la misma), una ideología cuya promesa de libertad individual no se realizaría (Kedourie, 1966), una identidad producida por los nacionalismos y al servicio del desarrollo económico (Gellner, 1988), o discursos políticos a disposición de las élites en su lucha por el poder (Breuilly, 1990; Brass, 1991). Es cierto que, desde la década de 1990, ha habido importantes contribuciones que han tratado de mostrar la viabilidad del nacionalismo, sea ello por su compatibilidad con el liberalismo (Tamir, 1993), tratando de dejar atrás el vínculo o el binomio nacionalismo/autoritarismo-fascismo, sea por sus positivos efectos en un mundo más globalizado (Calhoun, 2007). Sin embargo, creemos que, a pesar de esas aportaciones, la insatisfacción con el nacionalismo continúa ${ }^{7}$, y asimismo pervive con fuerza su éxito político, y es a los insatisfechos a los que les gustaría encontrar una solución que consiguiese «superar» el nacionalismo. Aquí está el problema, o, dicho en forma de pregunta, "¿es posible escapar del nacionalismo?».

Nuestra respuesta es «no». ¿Por qué? En este artículo, proponemos que el nacionalismo no es una ideología entre otras, sino la que ha estado en la base de la constitución de los estados desde el período revolucionario que, a finales del siglo XVIII, llevó a la «soberanía nacional», tanto en los Estados Unidos

4. Una interesante revisión sobre las consecuencias de las dos guerras mundiales en el pensamiento sobre el nacionalismo puede ser encontrada en Lawrence (2005). Asimismo, una importante contribución al entendimiento de la ambigüedad del nacionalismo en el marco del debate sobre el cosmopolitismo está contenida en un libro de Calhoun (2007) de reciente publicación.

5. Un texto que refleja con claridad la relación que comúnmente se establece entre nacionalismo y violencia es el de Visvanathan (2006), el cual está plagado de referencias en este sentido.

6. A este respecto, puede tomarse como texto de referencia el Manifiesto del partido comunis$t a$, de Marx y Engels (1971), texto cuyo original es de mediados del siglo XIX.

7. Pervive una importante resistencia a aceptar que el nacionalismo es compatible con el liberalismo (o la democracia liberal). Un ejemplo palmario de esta preocupación es el artículo de Laegaard (2007). 
como en Francia ${ }^{8}$. Esto significa que rechazar el nacionalismo tiene dos implicaciones importantes, sobre las cuales no parece que haya habido una reflexión particularmente extensa y profunda en el pasado entre sus críticos. Por un lado, supone hacer frente a la refutación del principio sobre el que se constituyen la mayoría de los estados contemporáneos, sobre todo los de tipo democrático. Por otro lado, implica que tenemos capacidad intelectual para sugerir y estamos dispuestos a aceptar a otro tipo de soberano distinto a la nación o el pueblo.

\section{II. ¿Cómo se legitima la existencia de los estados?}

En el sentido weberiano, toda forma de dominación (o autoridad) tiene que estar fundamentada en un principio de legitimación, lo que produce diferentes tipos de gobierno y de aceptación del mismo (Weber, 2007). Previo a tomar cualquier decisión sobre la forma de gobierno, está la definición del conjunto social que será gobernado 9 . El principio de nacionalidad tiene ese propósito, y sólo un acuerdo sobre el mismo produce sociedades nacionalmente cohesionadas.

El nacionalismo es una ideología y, en términos generales, un discurso disponible. Y es algo más. Se ha convertido en un principio jurídico previo a la propia legitimidad del poder y de la legalidad que del mismo emerge. Este principio, tanto político como jurídico, tan comúnmente proclamado como sustento de las democracias liberales, ha dado lugar a la existencia de estados constitucionales, en sentido estricto, y con seguridad a todos o casi todos los demás, en un sentido más lato. Los estados se legitiman como institucionalidad al servicio de las naciones, lo que sitúa a la nación en el centro de la argumentación política. El principio de nacionalidad no es un principio retrógrado, sino progresista, pero es un principio vacío, porque el acuerdo sobre el mismo tiene un impacto insatisfactorio en la realidad (piénsese, por el contrario, en el principio de protección de los derechos humanos).

La soberanía nacional es el producto de pensar las comunidades políticas como naciones. Esto no significa que las naciones aparezcan históricamente antes que los nacionalismos, ni necesariamente lo contrario, cuestión que, en todo caso, tampoco ha tenido una solución definitiva en la literatura sobre el origen de naciones y nacionalismos ${ }^{10}$. La nación es previa desde un punto de

8. Anderson (1991) subraya que este principio triunfó por primera vez en América, en el marco de los procesos de descolonización que allí tuvieron lugar entre finales del siglo XVIII y principios del XIX. En particular, destaca los casos de Hispanoamérica, que, habiendo precedido a la mayoría de los europeos, han tenido menos visibilidad que éstos en los estudios sobre el nacionalismo.

9. A este propósito, puede leerse a Greenfeld (1999), cuando habla de la democracia.

10. Esta incapacidad para alcanzar un acuerdo entre los académicos en torno al origen de las naciones queda bien reflejada en la extensa reflexión que Smith (1998 y 2001) dedica a los paradigmas, el tratamiento de los cuales indicaría que las teorías ofrecidas se agruparían de acuerdo con los supuestos de partida que comparten, los que finalmente se corresponderían 
vista jurídico-político, pero nada más. Los que transforman esta abstracción en un relato histórico se equivocan con mucha frecuencia. En todo caso, el hecho de que se haya ido estableciendo la exigencia o el requisito político de razonar desde la nación y a partir de su presencia, voluntad y necesidades, es un hecho incuestionable en la modernidad postrevolucionaria y en la historia constitucional que la ha acompañado ${ }^{11}$. La nación es la verdadera vencedora en las luchas ideológicas y en los conflictos políticos, y por eso no debe extrañar que el nacionalismo sea su justo alter ego, ampliamente victorioso y que llega al siglo XXI con la fuerza de su comienzo, e incluso es posible que más. Pero esto no es sólo motivo de debate ideológico, y puede quedar perfectamente ilustrado, como ya adelantamos, en los textos jurídico-políticos más importantes sobre los que se han instituido crecientemente los estados desde finales del siglo XVIII: las constituciones. En este sentido, observamos como la soberanía nacional opera en múltiples lugares y momentos, lo cual llega a ser un acontecimiento políticamente clave, y mostramos algunos ejemplos significativos a continuación al referirnos a textos constitucionales, o que tienen este carácter, surgidos a partir de la independencia de los Estados Unidos, sucedida a fines del siglo XVIII.

We the People of the United States [...] do ordain and establish this Constitution for the United States of America. (Preámbulo de la Constitución de los Estados Unidos de 1787)

La soberanía reside esencialmente en la Nación. (Artículo $3^{\circ}$ de la Constitución española de 1812)

La soberanía nacional reside en el pueblo español. (Artículo 1, punto 2, de la Constitución española de 1978)

La Souveraineté est une, indivisible, inaliénable et imprescriptible. Elle appartient à la Nation. (Artículo 1, del título III, de la Constitución francesa de 1791)

La souveraineté réside dans le peuple. (Artículo 25 de la Constitución francesa de 1793)

La soberanía reside esencialmente en la Nación. (Artículo $5^{\circ}$ de la Constitución de Chile de 1980)

con explicaciones diferentes. Esta situación de la investigación podría ser interpretada como un punto de llegada, al cual Smith responde con su identificación con el paradigma etnosimbólico y no con una aproximación con aspiraciones de integrar las aportaciones de los diferentes enfoques.

11. En este punto, nosotros optamos por tratar de restringir nuestras reflexiones al ámbito político, frente a las afirmaciones más omniabarcantes de Greenfeld (1999). En este sentido, ella afirma que «el nacionalisme representa el marc cultural de la modernitat, el mitjà cognitiu que dóna forma a la manera com percebem la realitat, està necessàriament i centralment relacionat amb la nostra construcció dels seus aspectes socials, politics i culturals en el sentit més estricte» (1999: 11). 
Los estatutos de autonomía aprobados en España en el marco definido por la Constitución española de 1978 mantienen la misma lógica, si bien con diferentes énfasis políticos. Seguidamente podemos observar algunos casos particularmente notables.

El Pueblo Vasco o Euskal Herria, como expresión de su nacionalidad, y para acceder a su autogobierno, se constituye en Comunidad Autónoma dentro del Estado español bajo la denominación de Euskadi o País Vasco. (Artículo 1 del Estatuto de Autonomía del País Vasco de 1979)

Os poderes da Comunidade Autónoma de Galicia emanan da Constitución, do presente Estatuto e do Pobo. (Artículo 1, punto 3, del Estatuto de Autonomía de Galicia de 1981)

Artículo 8. Los poderes de la Comunidad de Madrid se ejercen a través de sus instituciones de autogobierno: la Asamblea, el Gobierno y el Presidente de la Comunidad.

Artículo 9. La Asamblea representa al pueblo de Madrid, ejerce la potestad legislativa de la Comunidad, aprueba y controla el Presupuesto de la Comunidad, impulsa, orienta y controla la acción del Gobierno. (Estatuto de la Comunidad de Madrid de 1983)

1. La Generalitat es el sistema institucional en que se organiza políticamente el autogobierno de Cataluña.

4. Los poderes de la Generalitat emanan del pueblo de Cataluña y se ejercen de acuerdo con lo establecido en el presente Estatuto y la Constitución. (Artículo 2 del Estatuto de Autonomía de Cataluña de 2006 ${ }^{12}$ )

Como complemento, nos referimos muy brevemente a dos tratados internacionales especialmente relevantes, en los cuales también encontramos claras referencias a las naciones y/o a los pueblos, lo cual nuevamente los sitúa como un elemento central en la argumentación política:

Artículo A: La Unión tiene su fundamento en las Comunidades Europeas [...] Tendrá como misión organizar de modo coherente y solidario las relaciones entre los Estados miembros y entre sus pueblos.

Artículo F, punto 1: La Unión respetará la identidad nacional de sus Estados miembros, cuyos sistemas de gobierno se basarán en los principios democráticos. (Tratado de la Unión Europea de 1992, comúnmente conocido como Tratado de Maastricht)

12. Para nuestros propósitos, poco importa que este estatuto haya sido recurrido ante el Tribunal Constitucional. Independientemente de la resolución que eventualmente éste pueda adoptar, la lógica del principio de nacionalidad puede observarse con claridad, lo cual es el motivo último de esta exposición. 
Nosotros los pueblos de las Naciones Unidas. (Preámbulo de la Carta de las Naciones Unidas de 1945)

Como adelantábamos en la introducción, si no estamos satisfechos con el principio de nacionalidad, deberíamos estar en condiciones de sustituirlo. Desafortunadamente para los insatisfechos, tal sustituto todavía está por llegar. Mientras tanto, cabe decir que es posible descubrir un gran consenso a favor del principio mencionado cuando pensamos en algunas alternativas que han tenido presencia en la historia política de la humanidad. Hoy en día, pocos aceptarían como fuentes de legitimidad del gobierno a la palabra de Dios o el derecho divino, la tradición o las monarquías hereditarias, y la misma suerte han corrido los regímenes políticos que actuaban en nombre de la clase social (clase obrera o proletariado). Destronar a la nación significa entronizar a otra idea legitimadora. A los insatisfechos, les preguntaría "¿cuál o qué combinación de cuáles?»o "¿qué alternativa proponen?».

\section{III. ¿Qué es el nacionalismo?}

El objetivo de este escrito no es entrar en disquisiciones sobre la definición del concepto de nacionalismo. En particular, Smith (2001) habla de tres sentidos particularmente relevantes de esta idea, y nosotros prestamos atención a uno de ellos: el nacionalismo como ideología. Los otros dos son el entendimiento del mismo como movimiento político o como un lenguaje simbólico. Creo que ninguno de estos dos tiene que ver con la emergencia del nacionalismo como "problema» político en la época posterior a la Revolución Francesa. El nacionalismo como discurso surge en el marco de una realidad política que respondió dialécticamente a un principio de legitimidad que se consideró obsoleto, el principio monárquico, el cual era una forma de legitimidad tradicional, por seguir la terminología de Weber (2007), la cual ponía en primer plano el derecho al trono o al gobierno alcanzado por las vías de herencia históricamente legítimas. En otras palabras, no cualquiera podía reinar, y eso había servido para reducir los conflictos entre los señores feudales que estallaban por razón de la sucesión. No importa que la revolución mencionada se iniciase sin la perspectiva de un destronamiento, sino que en el contexto político que propició se fue imponiendo otro soberano, la nación, y otro principio de legitimidad, el de nacionalidad. Poco importa que la nación comenzase siendo un ente abstracto, más que concreto; socialmente excluyente, más que incluyente, o un producto principalmente intelectual, más que algo sentido por la población francesa a la sazón. Por primera vez, el gobierno, y con ello la legalidad, se constituye a partir de la soberanía de la nación. Desde un punto de vista ideológico, esto provocará que la definición y, con ello, la identificación de la misma pase a ocupar una posición central en los debates políticos posteriores, siempre y cuando se aceptase al nuevo soberano o, en otros términos, el nuevo principio de legitimidad. Esto conducirá a que la generalizada aceptación de este principio convierta en una falacia la distinción entre nacionalistas y no nacio- 
nalistas. Todos serán nacionalistas de algún modo. El contrario del nacionalista no será el no nacionalista, sino, teóricamente, el que continúe defendiendo otras formas de soberanía, como será el caso de la monárquica o la divina. No obstante, históricamente, incluso en estos casos la apelación a una nación será un lugar común.

Por tanto, tratamos el nacionalismo como una ideología que propugna que el soberano es la nación. Proponemos que ha sido generalmente aceptada en el mundo posterior a la Revolución Francesa, y de hecho es con frecuencia parte de las ideologías postrevolucionarias. Además, creemos que seguirá operando en el futuro próximo e incluso lejano, pues el internacionalismo se ha instituido sobre la base del reconocimiento del principio de la nacionalidad, y existen abundantes ejemplos de movimientos que siguen promoviendo sus intereses con el vocabulario, ya bien conocido y manido, del pueblo y la nación ${ }^{13}$.

Para reforzar estos argumentos, es particularmente interesante prestar atención a las siguientes afirmaciones de Anthony D. Smith (2001), realizadas cuando presenta el que denomina paradigma modernista:

Such planned «nation-building» was essentially a modern process, which found no real parallel before 1789. There had, it is true, been welfare programmes of enlightened monarchs in previous epochs, great construction projects, and even the large-scale «modernization» of administration [...] But there was no collective design to their work; it was conducted in the name of, and on behalf of, an individual or a ruling class. Nor was there any ideology of the sovereign people sharing a common history and culture, to whom supreme loyalty was owed and for who great sacrifices must be made. There was no question in earlier epochs of mobilizing the people to participate in politics at the centre, nor of the need for men, let alone women, to become politically aware and active «citizens». (Smith, 2001: 45)

As a process of «nation-building» and as an ideology and movement, nationalism and its ideals of national autonomy, unity and identity, are relatively modern phenomena, which have placed at the centre of the political stage the sovereign, united and unique nation, and have made over the world in their image. (Smith, 2001: 46)

Smith, por tanto, reconoce la modernidad de los nacionalismos, pero, no obstante, en su obra insiste en afirmar que una explicación de los mismos tiene que retrotraerse a las identidades prerrevolucionarias, y no puede fundamentarse sola o principalmente en las condiciones propias de la «modernidad».

13. Es interesante observar como, en las reflexiones sobre el multiculturalismo (Kymlicka, 2001), emerge de una manera peculiar la soberanía nacional en la forma de lo que podríamos denominar un "principio de culturalidad", de acuerdo con el cual todo conjunto poblacional que comparte una cultura debería disfrutar de cierto grado de autonomía para su autogobierno dentro de la comunidad política mayor. El principio de culturalidad sería un principio de nacionalidad suavizado con el propósito de favorecer la convivencia de una forma relativamente pacífica con el hecho de vivir en una sociedad intercultural. 
En todo caso, además de entender que el nacionalismo puede ser comprendido como una ideología que promociona el principio fundamental sobre el cual se ha constituido la modernidad política, al menos en Occidente, el esfuerzo de romper la asociación tan consolidada, y automatizada, entre este fenómeno y los conflictos políticos, requiere que desarrollemos nuestro argumento abordando otras dimensiones que también han estado en la base de las argumentaciones sobre las que se basan dichos razonamientos. Ése es el sentido de los siguientes apartados.

\section{El relato sobre el surgimiento del nacionalismo}

\section{IV.a. El surgimiento}

Si entendemos el nacionalismo como ideología, tal y como hacemos aquí, encontramos en los trabajos sobre su origen, también en la obra de Anthony D. Smith (y decimos "también» puesto que, frecuentemente, él retrotrae las causas de su emergencia a un período anterior a los acontecimientos revolucionarios que tuvieron lugar en Estados Unidos y Francia a fines del siglo XVIII ${ }^{14}$ ), que se explica su surgimiento como una combinación de dos procesos. Por un lado, se enfatiza el papel de la nación soberana que nace con las revoluciones mencionadas y, por otro lado, la aparición de lo que podríamos llamar el «cultural-nacionalismo» alemán (Guibernau, 1996), visión que proponía una definición de la identidad de la nación germana a partir de rasgos étnicos, principalmente la lengua (Fichte, 1988). Sobra decir que en ningún caso hablamos de naciones democráticas, sino sólo de naciones. La ideología nacionalista sería el producto de la combinación de nación soberana y cultura. Sin embargo, nosotros consideramos innecesario incluir el segundo momento en la etiología de la ideología nacionalista, pues el nacionalismo ya había tomado forma en las revoluciones estadounidense y francesa. Por ejemplo, en Francia, la leva en masa sólo pudo justificarse incluyendo al individuo en un cuerpo social más amplio a cuyo destino aquél debía adaptarse. La Francia napoleónica fue, en lo que tuvo de continuadora de la Revolución, expresión del primer nacionalismo, la ideología puesta en acción, y con consecuencias bien conocidas que dieron lugar al Congreso de Viena (1814-1815), en el cual las dinastías imperiales europeas a la sazón intentaron poner fin a la doctrina nacionalista ${ }^{15}$.

14. De un modo más matizado, Smith resume su propia posición de la manera siguiente: «My own analysis is concerned with a third problem: the nature and role of nations in history. While acknowledging the modernity of nationalism, the ideology, movement and symbolism, and the recent formation of most nations, I have become interested in the possibility of nations prior to nationalism, at least in a few cases, and its implications. But, in general, my approach has focused on the way that prior, and often premodern, ethnic ties and ethnies have influenced, and in some cases formed the basis for, subsequent nations and nationalisms» (Smith, 2001: 60).

15. En la literatura académica sobre el origen del nacionalismo, será habitual contraponer teorías cuyos conceptos de partida son diferentes, y ello ha sido fuente de confusión y falsos 
El nacionalismo nace con la instauración revolucionaria del principio de soberanía nacional. Ello llevó a un cuestionamiento lógico sobre quiénes componían el cuerpo nacional. No se discute el nacionalismo, ampliamente aceptado, sino las definiciones de la nación y sus efectos, lo que es una cuestión muy diferente. La primera, la aceptación del nacionalismo, es lógicamente previa. En cualquier caso, tratándose el nacionalismo de una ideología que promueve la soberanía antedicha, sus orígenes no debiesen ser buscados ni en la identidad (Smith, 1986), ni en el culturalismo alemán o el romanticismo. El nacionalismo es un hijo natural, antes de nada, del liberalismo ${ }^{16}$. No existe, por tanto, y es otra idea que hay que destacar, una oposición entre liberalismo y nacionalismo (más que le pese a críticos del nacionalismo como Lord Acton). Son complementarios. El liberalismo limitó los poderes del monarca soberano, o le privó de los mismos, y los transfirió a la nación o al pueblo. El nacionalismo potenció este hecho definiendo lo constitutivo de esa nación y promoviendo su soberanía política. No obstante, posteriormente, el nacionalismo mostraría su adaptabilidad como principio y formaría parte de complejos ideológicos que lo incluirían de forma explícita (p. ej.: fascismo y nacionalsocialismo) o lo darían por supuesto (p. ej.: las liberaldemocracias contemporáneas). De este modo, se podría afirmar que el nacionalismo es una "consecuencia imprevista» (Weber, 2002) del éxito del liberalismo y del paralelo debilitamiento y extinción progresiva del principio de soberanía monárquica.

Ha sido históricamente evidente la insistencia del nacionalismo en la libertad, si bien la del individuo se vehiculaba a través de la comunidad de pertenencia. En sentido estricto, y de acuerdo con su capacidad para incorporar la voz de los individuos en la configuración de la voluntad del soberano, podríamos hablar de dos tipos de nacionalismos. El primero, el inicial, de directa descendencia liberal, enfatizaría la soberanía nacional, pensando la nación como un ente abstracto, una voluntad colectiva sin relación necesaria con la suma de las voluntades individuales. El segundo, el que acompañó a la formación de las liberaldemocracias, dio pábulo a la idea de soberanía popular o del pueblo, la cual debía generarse de abajo a arriba, y ser una expresión de las voluntades particulares libremente expresadas. En realidad, en contra de sus insistentes manifestaciones, la soberanía popular de los regímenes comunistas fue del primer tipo ${ }^{17}$. Podríamos, entonces, distinguir entre dos tipos de nacionalismo: el nacionalismo nacional y el nacionalismo nacionalpopular.

debates. La cuestión de su origen es particularmente importante dentro de las aportaciones sobre el nacionalismo, porque, en base a la respuesta que se da a la misma, se ha configurado la clasificación de paradigmas y teorías con mayor aceptación (Smith, 1998; Llobera, 1999; Özkirimli, 2000). Para un principio de crítica de estas tipologías, puede verse Gayo, 2000 y 2002.

16. Greenfeld (1999) vincula el nacionalismo primeramente con la democracia. Sin embargo, parece más exacto históricamente asociar el primero al liberalismo. La relación entre el nacionalismo y la democracia no siempre ha sido fácil.

17. Aunque por ser el pueblo sólo una parte del colectivo social, deberíamos, en este caso, hablar de «soberanía de clase» (o de la clase obrera). 


\section{IV.b. Las consecuencias: nacionalismos civico y cultural ${ }^{18}$}

Si la primera versión del nacionalismo, la que partió del reconocimiento de la soberanía nacional, sirvió para justificar el destronamiento de Luis XVI y la política expansionista de Napoleón, la segunda versión, la construida, sucesivamente, por Herder y Fichte, prestó respaldo a la identificación de Alemania $y$, posteriormente, a la unificación bismarckiana, producto de un prolongado, y no siempre pacífico, proceso que tuvo lugar durante buena parte del siglo XIX. De aquí emerge la distinción, o el principio de la misma, desde un punto de vista más contemporáneo, entre los conocidos como nacionalismos cívico y cultural. Por tanto, vemos que, independientemente de la mayor o menor verdad de esta clasificación, los nacionalismos emergentes respondieron a una lógica histórica dentro de la cual se mostraron funcionales a ciertos propósitos, y no fueron el derivado de una reflexión sobre la nación en el vacío o de manera pura o principalmente abstracta, y menos una simple constatación histórica. En otros términos, los nacionalismos fueron una nueva manera de verbalizar y articular intelectualmente, para su promoción, intereses que ya existían, si bien llevó a consecuencias imprevistas. Por tanto, la distinción entre los nacionalismos cívico y étnico es en buena medida espuria, sobre todo si queremos pensarla como un epifenómeno de un supuestamente preexistente ethos nacional que está llamado, más tarde o más temprano, a ser protagonista de la historia humana.

\section{V. ¿Es tan peligroso el nacionalismo?}

Si todos los estados se legitiman a partir del principio de nacionalidad, o aspiran a hacerlo, y no siempre se habla de nacionalismo, parece que no debiera ser la nación y el nacionalismo presente en una doctrina legitimadora la fuente de los conflictos que se le achacan. El nacionalismo no es ni más ni menos disruptivo que otras doctrinas cuando se quieren llevar a la práctica ${ }^{19}$. El nivel de conflicto dependerá de las circunstancias en las que trate de ser aplicado. Eso quiere decir que la misma ideología puede ser considerada benéfica y salvadora en un momento, y desastrosa en otro. En otros términos, el nacionalismo no es malo o bueno en sí mismo, como doctrina de carácter general, sino que normalmente ha sido adjetivado de acuerdo con las circunstancias en las que ha actuado y los propósitos que lo han animado.

En todo caso, es claro que hay que distinguir entre la ideología nacionalista, de hecho muy extendida como principio de legitimación, como quedó establecido, y el uso que se realiza de la misma. Esto es importante, porque la ideología no siempre lleva al uso, sino que la práctica política muy frecuentemente conduce a su empleo. Lo que esto significa es que no es el nacionalismo

18. Para una crítica de esta distinción, véase Máiz (2004).

19. Piénsese en las revoluciones liberales en los albores de la modernidad, o las revoluciones socialistas mucho más recientes y que todavía alcanzamos a recordar, al menos por sus efectos. 
el origen del problema, sino que a menudo es el problema el que da lugar al nacionalismo. Diagnosticar la causalidad es fundamental, pues, de otro modo, no sabríamos qué hacer para dar solución a o entender el conflicto planteado. Distingamos entre las dos versiones.

A la primera versión, podríamos denominarla idealista, y es la que con mayor frecuencia está en uso. Brevemente, el nacionalismo es una ideología que orienta la acción política de los individuos. Cambiando la primera, de forma más o menos automática, provocaríamos modificaciones en la segunda. Esta perspectiva justifica todo el debate sobre la ideología nacionalista y los ingentes y costosos esfuerzos, todavía en curso, para su superación.

La segunda versión podría ser llamada política, y suele insistirse en ella o bien como algo del pasado, o bien como una consecuencia de la primera versión. En resumen, se trata de que son los conflictos políticos, ya sea entre élites, ya sea entre diferentes comunidades, los que importan y estarían en la base del uso, estratégico, de la ideología nacionalista ${ }^{20}$. Lo que importa no está en la ideología, sino más allá de ella. Por tanto, la resolución del conflicto «ideológico» pasa necesariamente por enfrentar las bases políticas del mismo.

Es importante la modalidad que estamos utilizando, porque la versión política deja a la ideología o a las creencias en un segundo plano, la religiosidad con la que parecen comportarse algunos políticos (Smith, 2003), y pone en primer plano el problema político en toda su crudeza, lo que permite una discusión y una evaluación más racional. En otras palabras, desde este punto de vista, los políticos no pueden seguir ocultando sus razones, estrategias e intenciones detrás del manto ideológico que otorga la defensa de la «sagrada» nación.

\section{VI. ¿Cuándo molesta el nacionalismo?}

Básicamente, el nacionalismo molesta cuando quiere cambiar el mapa político, y esto se produce a través de dos mecanismos principales: la división y la expansión. En los casos en los que el nacionalismo está latente o es parte de la vida cotidiana, como el aire que se respira, parece no existir y por ello no es motivo de atención o conflicto ${ }^{21}$. Muere eventualmente como arma política.

Dicho en términos fenomenológicos, esta ideología forma parte, o da forma, de lo que consideramos natural en nuestro mundo. Ha sido interiorizada hasta tal punto que sólo nos parece discutible su negación. Es parte de lo que nosotros somos, y su negación, por ello mismo, es la nuestra, lo cual consideramos inaceptable. Ni siquiera podemos decir que aceptemos el nacionalismo porque nos guste, sino porque no hay alternativa viable que un hombre actual

20. Díez Medrano (2000) muestra como los nacionalistas vascos y catalanes no solamente recurrieron de forma estratégica al «nacionalismo», sino que, además, lo hicieron animados por conflictos distintos. La clave del entendimiento de éstos, en esta lógica, debería estar ligada con los últimos y no con el primero, es decir, la ideología nacionalista.

21. Para un tratamiento de la vivencia naturalizada y cotidiana del nacionalismo, ha devenido un clásico el libro de Billig (1995). 
pueda sostener sobre bases racionales. Nos pueden convencer de que la nación es otra, pero no de que no existe, puesto que incluso su negación, en los términos de una comunidad puramente imaginada (Anderson, 1991), si bien intelectualmente aprehensible, es poco creíble en nuestra experiencia de vida. ¿Quién no recurre a la cultura, al nosotros, al origen, para dar una justificación de su ser? ¿Quién no habla del otro, de lo ajeno, de lo que no nos pertenece, como parte de una alteridad que, finalmente, en forma de espejo, nos permite observar reflexivamente lo que somos y pensar en ello, incluso de forma crítica? En realidad, el nacionalismo como algo negativo nunca es el nuestro. El nacionalismo molesta cuando es el de otros, y sobre todo cuando pone en cuestión nuestra forma natural de ver el mundo.

Muy comúnmente, por lo tanto, el nacionalismo no es motivo de preocupación, porque no se percibe. Habitualmente molesta cuando le da cobertura ideológica a procesos de expansión o secesión territoriales, cuando, en definitiva, está ligado a una alteración del mapa político. Es en estos casos cuando se habla de nacionalismo. En este sentido, el ejercicio intelectual de identificar períodos de auge o emergencia de este fenómeno (Hobsbawn, 1992) olvida que siempre ha estado presente. Al estar inserto en la lógica de la modernidad política, la pregunta no debe ser producto de una sorpresa por su presencia, del tipo "¿por qué tenemos nacionalismo?» o "¿por qué de nuevo?», sino que debería causarnos asombro su desaparición, su silencio o su aparente agotamiento. La pregunta sería "¿cómo puede ser que no tengamos nacionalismo?», "¿qué fuerzas lo combaten?» o, finalmente, «¿por cuánto tiempo serán efectivas esas fuerzas?».

\section{Las soluciones políticas al nacionalismo}

En general, las soluciones políticas al nacionalismo no son una manera de superarlo, sino, por el contrario, de fortalecerlo e institucionalizarlo, y, en último extremo, de satisfacerlo. Las soluciones confirman el principio nacional y robustecen el logro de objetivos por parte de la ideología nacionalista, aunque no es infrecuente que debiliten la causa. Esto refleja perfectamente como, a menudo, el problema no es ideológico, para el cual no cabe pragmatismo en la solución, sino político, del tipo que sea. Obviamente, una solución política al nacionalismo sería superar el principio de nacionalidad, pero, como ya adelantamos previamente, esto plantea a su vez otro problema, que es el de su sustitución.

Las conocidas soluciones políticas al nacionalismo, en sus diferentes versiones (federalista, confederal, estado autonómico, y otras que eventualmente puedan caber dentro de este rubro) (Máiz, 1997), han sido soluciones políticas, más o menos exitosas en cada caso, a un conflicto político, pero no han permitido cerrar la cuestión nacional. Muy por el contrario, las soluciones han confirmado el principio de nacionalidad y han mostrado como en la modernidad, sea ésta políticamente democrática o no, el nacionalismo se ha convertido más que en una ideología de naturaleza similar a las otras alternativas, en 
una forma de comprender la constitución de comunidades políticas y de razonar sobre las formas de gobierno que es transversal a todas las ideologías y lugares. Y eso es así incluso cuando la legitimidad viene de Dios, como es el caso de las repúblicas islámicas, pues también para ellos hay un pueblo elegido que debe obedecer su ley, la cual no se reduce a meros principios abstractos sin conexión alguna con una realidad social concreta.

¿Por qué han fracasado las soluciones político-institucionales al conflicto nacional? Hay varios tipos de razones que pueden ser esgrimidas, pero aquí ofreceré dos que me parecen igualmente relevantes. La primera es ideológica: independientemente de la voluntad momentánea de los actores políticos que alcanzan acuerdos puntuales de convivencia, el principio de nacionalidad sigue operando, con su lógica implacable, y es una cuestión de tiempo que vuelva a salir a la luz reivindicando ser satisfecho. La segunda razón se origina en una comprensión deudora de la teoría de juegos. Normalmente, el uso de esta teoría ha implicado la selección de un juego para comprender una situación concreta en la que individuos o actores deben tomar decisiones en un escenario con grados variables de incertidumbre. Para nuestros propósitos, vale decir que el fracaso de las soluciones institucionales es debido a una falta de comprensión del juego que está en curso y de la lógica de la actuación de los actores. Brevemente, el conflicto nacional no es un juego estático, sino que, muy por el contrario, se trata de un juego dinámico en cuyo interior se desarrollan juegos estáticos momentáneos de los cuales se derivan acuerdos que nunca serán definitivos hasta que el principio de nacionalidad, según el entendimiento del mismo que tienen los actores, alcance su objetivo último, la coincidencia entre nación y estado, es decir, la soberanía nacional.

Sea como fuere, la que no cabe como solución es la que ha consistido en denunciar las inconsistencias lógicas del nacionalismo (Rodríguez Abascal, 2000). Esta aproximación parte de un supuesto que es falso y que, por tanto, invalida el interesante esfuerzo posterior. Se supone que el nacionalismo es una ideología como las otras (fascismo, comunismo, etc.) y que, en consecuencia, se puede ser nacionalista o no nacionalista. Sin embargo, en la realidad político-ideológica de la modernidad postrevolucionaria no cabe ser «no nacionalista». Discutir el nacionalismo es refutar el entendimiento sobre el que se han constituido, y siguen constituyéndose, las instituciones políticas contemporáneas más relevantes, los estados, al igual que gran parte de las organizaciones interestatales y supraestatales.

\section{Notas sobre el caso español}

Si tomamos en consideración lo previamente expuesto, nos encontramos con dos ideas incómodas que tenemos que extraer. En primer lugar, los llamados nacionalismos periféricos no se debilitarán por muchas concesiones que se les hagan, pues la lógica del principio de nacionalidad seguirá operando. Por tanto, la superación de los mismos no llegará con su fomento, sino con 
la promoción de una identidad distinta, a lo cual el Estado español parece haber renunciado hace ya un par de décadas ${ }^{22}$. Treinta años después del inicio de la transición, afirmar que el "problema» nacional se ha resuelto en España a través de la constitución del Estado de las Autonomías no puede ser una constatación histórica, y debe ser ubicado en el ámbito de los deseos incumplidos. Esta es una conclusión particularmente problemática, pues la Constitución española de 1978, principalmente en su título VIII, referido a la estructura territorial, se presentó como una solución histórica a las tensiones territoriales que se habían vivido en España. Si constatamos que, tres décadas más tarde, el conflicto continúa vivo, incluso de forma creciente, eso demostraría que la solución descentralizadora, al menos la que se llevó a la práctica, fracasó, conclusión que dejaría sin asidero gran parte del proyecto político que ha guiado la política de los gobiernos españoles durante la etapa democrática posterior a la dictadura franquista. Todos los estatutos y la problemática que les acompañó no son más que una extensión de la misma cuestión. No parece que los políticos que han protagonizado todo el proceso estén dispuestos a aceptar su fracaso, sabiendo además que la falta de orientaciones alternativas llevarían al país a un marasmo de impredecibles consecuencias.

En segundo lugar, aunque rige el principio de nacionalidad, todavía opera, lo cual se elevó a mandato constitucional, el principio monárquico o de legitimidad dinástica. España es una monarquía, eso sí constitucional, y el rey representa la unidad de la nación española. Esto es importante, porque tiene un contenido histórico fuerte, lo cual molesta profundamente a los nacionalismos alternativos al español. Y eso es así por una razón: la lógica de razonamiento nacionalista privilegia al pasado y, en concreto, a la historia; y no importa aquí que, frecuentemente, los relatos sobre el origen y el desarrollo nacional sean poco verosímiles o escasamente fieles a lo realmente sucedido. Esto explica el énfasis que los nacionalistas "periféricos» han puesto a menudo en destruir los símbolos reales y todo aquello que tenga que ver con la figura del monarca ${ }^{23}$. En otros términos, si la existencia de España como nación está ligada a la presencia de la monarquía, la cual reinaría sobre todos los territorios o regiones del Estado, su desaparición, en esta argumentación, dejaría sin algo común a ese conjunto de ciudadanos, momento a partir del cual sólo quedaría funcionando el principio de nacionalidad, que dejaría abierta la solución que promueven los nacionalistas periféricos, la secesión de los territorios que dicen representar y la extinción de España tal y como la conocemos.

22. Para una reflexión diferente, que insiste en la presencia permanente pero no reconocida por sus portadores, sobre el nacionalismo español, véase Taibo (2007).

23. Por poner un ejemplo reciente, el periódico $A B C$ recogía, en su edición del 14 de septiembre de 2007, el siguiente titular: «La Audiencia Nacional investiga a los autores de la quema de fotos de los Reyes en Cataluña», hecho que se produjo frente al ayuntamiento de Gerona promovido por «independentistas catalanes». 
Modesto Gayo Cal

\section{Finalmente, el nacionalismo y la identidad}

Este apartado fue pensado para responder a las reflexiones que piensan el nacionalismo desde las identidades «individuales» ${ }^{24}$. En el caso español, una aportación reciente de este tipo se convirtió en libro bajo el título Identidades proscritas, escrito por Juan Pablo Fusi (2006) ${ }^{25}$. Si bien creo que son reflexiones valiosas, no atacan al centro de la cuestión. El nacionalismo no es un fenómeno que pueda ser enteramente comprendido desde la esfera individual. Se trata de un discurso ideológico que los individuos interiorizan sin que su constitución o producción esté a su disposición. El nacionalismo no es del individuo, al menos no en primera instancia, sino parte de una lógica política que lo supera y por la cual no podemos ni debemos responsabilizarlo. Atacar el nacionalismo desde la identidad de los individuos supone enfrentarlo desde la consecuencia y no desde la causa. Es, por tanto, una tarea desde el principio imposible y llamada al fracaso. En términos médicos, de un mal diagnóstico se derivará un tratamiento con escasos efectos y un pronóstico errado. Del presente trabajo, puede derivarse que el nacionalismo continuará independientemente de las voluntades individuales y su futuro será promisorio.

Entender el nacionalismo desde la identidad es la visión propia de los que consideran que el primero es un tipo de religión que configura la mente de sus portadores (Smith, 2001). En esta visión, el nacionalismo pierde lo que tiene de estratégico y los seres humanos viven obnubilados por las tradiciones, los símbolos, los relatos de tipo mítico, la admiración por los héroes y el apego a la tierra madre, heredados de sus ancestros. Pero, sobre todo, el nacionalismo está pensado desde la nación, y convierte a la misma en un prerrequisito para la constitución del primero, cuando, históricamente, fue la instauración del principio de nacionalidad lo que tuvo como consecuencia la activación política de la nación tal y como ha sido conocida en la modernidad.

El hecho de que la identidad individual no sea central para una correcta comprensión de las bases sobre las cuales el nacionalismo ha crecido, no significa que no haya razones detrás de la preocupación por la identidad ${ }^{26}$. El argumento se cree que funciona normalmente a favor de los que se consideran o son considerados «nacionalistas». Sintéticamente, se arguye que las identida-

24. Las comillas se deben a que tal expresión, «identidad individual», parece un oxímoron, si estamos pensando en identidades nacionales.

25. Hay otro libro, también de publicación reciente, escrito por Josep M. Colomer (2006), cuya conceptualización es un ejemplo palmario de identificación de identidad, que le presupone de forma acrítica a Cataluña y, en general, a las que denomina «pequeñas naciones», y nación. Cabe la duda sobre la intencionalidad del autor, pero las implicaciones políticas de las tesis que promueve son evidentes en su apoyo al nacionalismo catalán. Aunque en este caso no queremos extraer conclusiones precipitadas, la convivencia que históricamente ha existido entre la reflexión intelectual sobre el nacionalismo y el papel de los intelectuales en contextos políticos concretos, en los cuales a menudo abandonan toda pretensión de objetividad o imparcialidad, ha sido bien estudiada por Lawrence (2005).

26. Algunas notas sobre la importancia práctica de la reflexión teórica sobre el origen de las naciones puede ser encontrada en la introducción del artículo de Gayo (2000). 
des colectivas están en la base del sentimiento de comunidad que se traduce en la conformación de las naciones. La activación política de éstas últimas da lugar al nacionalismo, que es visto como la expresión moderna de identidades previamente constituidas, generalmente, a lo largo de un prolongado proceso histórico de diferenciación con respecto al exterior y de articulación significativa hacia el interior. En esta lógica argumental, si demostramos, como pensaría Fusi (2006), que las identidades previas al nacionalismo nunca estuvieron ahí, estaríamos plantando la semilla de una refutación pública de aquél. En esta aproximación idealista, lo central serían las ideas y no lo que ocurre en la sociedad en la que el fenómeno se da.

Decía que normalmente se considera que esta argumentación funciona a favor de los llamados "nacionalistas", o eso creen ellos y sus detractores, aunque sólo sea por el simple hecho de que les ha funcionado, cuando, lógicamente, con frecuencia debería haber mostrado su debilidad. En otros términos, si la nación reivindicada ha sido tan fuerte históricamente desde un punto de vista identitario, en los casos en los que tal comunidad ha carecido de soberanía política efectiva, ¿por qué ha sido así? O, si queremos, ¿̨por qué la movilización a favor de su soberanía ha tenido lugar en el escenario moderno? ¿Por qué no antes? Si existe una realidad sociohistórica caracterizada por fuertes identidades nacionales y débiles instituciones políticas, ello demuestra que el nacionalismo, incluso si pensamos que está íntima y constitutivamente ligado a la identidad, tiene que deber mucho a otro tipo de fenómenos. Aquí hemos destacado uno que no es puramente intelectual, aunque en su origen lo fuera: la instauración del principio de nacionalidad como base de legitimación de los estados contemporáneos. Es en este marco en el que se enciende el «fuego» nacionalista y no antes. La identidad no fue suficiente o, simplemente, nunca importó políticamente o jamás existió.

\section{Conclusiones}

Superar el nacionalismo implicaría superar el principio de legitimidad política sobre el que están constituidos los estados en la actualidad. Superar el nacionalismo, entonces, supone la aparición de otra fuente de legitimación. Cuando se plantean escenarios alternativos al nacional, sobre cuyo mapa político vivimos, deberían indicarse también los principios a partir de los cuales las comunidades políticas se configurarían como tales. Hemos visto que otras fuentes de legitimación política que han surgido en la historia hoy día no son ampliamente compartidas o serían de muy difícil aceptación.

Es fundamental entender que el nacionalismo es deudor, o una consecuencia, del principio de legitimidad política sobre el que se constituyeron los estados, de forma declarada o no, desde hace dos siglos, es decir, el principio de nacionalidad, el cual sitúa a la nación como la entidad políticamente soberana por excelencia. Anormalizar el nacionalismo significa rechazar este axioma y poner en duda las bases sobre las que se constituyeron y se asientan los estados contemporáneos. Además, esto supondría plantear la necesidad de pro- 
ducir intelectualmente otro que viniese a sustituir al anterior, con efectos no conocidos, o ya sabidos y por ello históricamente arrumbado. En un sentido diferente, normalizar el nacionalismo, y no tratar de superarlo, implica reconocer que el principio de nacionalidad no ha sido de fácil aplicación y ello ha traído como consecuencia que una evaluación del mismo por sus efectos produzca un resultado ambiguo. $\mathrm{Si}$, por un lado, ayudó decisivamente a trasladar la soberanía a manos de los gobernados, por otro, ha animado un sinfín de conflictos, pues el mapa político prenacionalista no se había conformado de acuerdo con el principio mencionado y la identificación de las naciones, lejos de ser una cuestión que podemos dar por supuesta, se ha mostrado como un asunto plagado de disputas políticas y conflictos sociales ${ }^{27}$.

El nacionalismo no es necesariamente, y probablemente no muy habitualmente, la causa de los conflictos nacionales. Muy por el contrario, éstos suelen tener su base en problemas sociales y tensiones políticas de índole distinta a la ideológica. En este sentido, el nacionalismo es un discurso disponible al que las élites políticas e intelectuales han recurrido a menudo para solventar disputas. Por ello el nacionalismo es un fenómeno tan variado, a disposición de todo tipo de ideologías, imposible de ser reconstruido por una sola teoría, pues las razones para su empleo han sido muy diferentes. Pensemos en el caso español. Los conocidos como «nacionalismos periféricos» españoles (el vasco, el catalán y el gallego), por poner los principales ejemplos, los más exitosos, tienen historias muy diferentes y han sido utilizados tanto por las derechas como por las izquierdas. En todos estos casos, difícilmente puede achacarse al nacionalismo la culpabilidad por lo que han hecho sus portadores oficiales. En buena medida, han sido conflictos entre las élites y procesos migratorios, los que condujeron al recurso al nacionalismo, y no fue éste el que llevó a los primeros.

Seguir pensando que el nacionalismo es un fenómeno únicamente propio de los que se declaran nacionalistas es un gran error y a menudo una herramienta intelectual para su descrédito. Si no se puede decir que la gran mayoría de los individuos son, consciente o abiertamente, nacionalistas, al menos es posible afirmar que en su mayor parte promueven inconscientemente una cierta forma de nacionalismo, al aceptar el principio de soberanía sobre el que se constituyeron «legítimamente» los estados en los que viven. En todo caso, el nacionalismo no se refiere principalmente a los individuos, sino a una ideología que sostiene, en último extremo, que debe haber una comunión o una identidad entre estado y nación, o, en otros términos, que toda nación tiene derecho a autogobernarse (autodeterminación). Esto es la soberanía de la nación, la cual se ha convertido, más que en una ideología, en un supuesto o principio implícito en la lógica que ha caracterizado hasta nuestros días a la modernidad política postrevolucionaria.

27. En este sentido, Visvanathan (2006) afirma que «Such a congruency between people, history and territory was easy at the level of definition but problematic at the level of reality» (p. 533). 
El drama de la modernidad política, donde gobierna el principio de nacionalidad, no es el de la presencia de los nacionalismos, sino que, en todo caso, viene provocado por la combinación de dos fenómenos con un potencial disruptivo similar y que con frecuencia han sido ensalzados, sobre todo dentro de las tradiciones de pensamiento de lo que comúnmente hemos identificado como la izquierda. Estos son el idealismo como forma de aproximación a la realidad social, cuya solidez (de la realidad) por ello mismo se evapora, y la revolución como método legítimo y adecuado para la transformación de la realidad sociopolítica, siempre de acuerdo con la visión idealista que está en curso en cada caso concreto.

El hecho de entender el nacionalismo desde la nación implica que la reflexión sobre el nacionalismo parte de un hecho: la constatación de su existencia. De la presencia incuestionable de la nación, tan cara a todo nacionalismo, se derivarían sus derechos políticos y una práctica política a la que no haría falta más que reivindicar lo que ya ha sido ampliamente reconocido y aceptado, el derecho de toda nación o comunidad nacional a autogobernarse. Este artículo parte de otra constatación. Históricamente, se ha podido observar que la identificación de las naciones no ha sido un proceso libre de polémicas. La importancia de identificar a las naciones no viene dada por su mera existencia, a menudo tan discutida, sino por el contexto que genera el reconocimiento del principio de nacionalidad en el período de nuestra modernidad política postrevolucionaria. Incluso si las naciones no existían, desde ese momento y bajo el nuevo contexto, había que buscarlas o simplemente inventarlas. El nacionalismo propone un ideal, al igual que las teocracias cuando dicen regirse por la ley de Dios. Este punto es crucial para entender los límites de su aplicación a la realidad y la necesidad de hacer uso del mismo con idéntica cordura y mesura a la que exige cualquier otro ideal.

Finalmente, la pregunta que debemos plantearnos no es si queremos o no nacionalismo, o «¿cómo podemos derrotarlo?». La pregunta debería ser «¿qué o cuánto nacionalismo queremos?». En este sentido, las comunidades políticas que ignoren la lógica sobre la que se constituyeron estarán sembrando la semilla de su futura desaparición.

\section{Referencias bibliográficas}

ANDERSON, Benedict (1991). Imagined communities: Reflection on the origin and spread of nationalism. Londres: Verso.

ARMSTRONG, John (1982). Nations before nationalism. Chapel Hill: University of North Carolina Press.

BiLlig, Michael (1995). Banal Nationalism. Londres: Sage.

BRASS, Paul (1991). Ethnicity and Nationalism. Theory and comparison. Nueva Delhi: Sage Publications.

BREuiluY, John (1990). Nacionalismo y Estado. Barcelona: Pomares-Corredor (1 $1^{\text {a }}$ edición en inglés, 1985).

Calhoun, Craig (2007). Nations Matter: Culture, History, and the Cosmopolitan Dream. Nueva York: Routledge. 
Carta de las Naciones Unidas (1945). Consultada en [http://www.un.org/spanish/aboutun/charter/index.htm]

Colomer, Josep M. (2006). Grandes imperios, pequeñas naciones. Barcelona: Anagrama.

Constitución de Chile de 1980. Consultada en [http://www.camara.cl/legis/constitucion/contitucion_politica.pdf]

Constitución española de 1812. Consultada en [http://www.constitucion.es/otras_constituciones/espana/txt/1812.txt]

Constitución española de 1978. Consultada en [http://www.constitucion.es/constitucion/lenguas/txt/castellano.txt]

Constitución de Estados Unidos de 1787. Consultada en [http://www.archives.gov/ national-archives-experience/charters/constitution_transcript.html]

Constitución francesa de 1791. Consultada en [http://www.conseil-constitutionnel.fr/textes/constitution/c1791.htm]

Constitución francesa de 1793. Consultada en [http://www.conseil-constitutionnel.fr/textes/constitution/c1793.htm]

Díez Medrano, Juan (2000). Naciones divididas. Madrid: CIS.

Estatuto de Autonomía de Cataluña (Ley Orgánica 6/2006). Consultado en [http://www.boe.es/boe/dias/2006/07/20/pdfs/A27269-27310.pdf]

Estatuto de Autonomía de Galicia (Ley Orgánica 1/1981). Consultado en [http://www.xunta.es/estatuto]

Estatuto de Autonomía de Madrid (Ley Orgánica 3/1983). Consultado en [http://www.madrid.org/pres_sgt/legislacion/leyes/estatutoautonomia.htm]

Estatuto de Autonomía del País Vasco (Ley Orgánica 3/1979). Consultado en [http://estaticos.elmundo.es/especiales/2005/06/espana/estatutos_autonomia/estatutos/pdf/e_pais_vasco.pdf]

Fichte, J.G. (1988). Discursos a la nación alemana. Madrid: Tecnos.

FUSI, Juan Pablo (2006). Identidades proscritas: El no nacionalismo en las sociedades nacionalistas. Barcelona: Seix Barral.

GaYo CAL, Modesto (2000). «Anthony D. Smith y Ernest Gellner: un debate en torno a la modernidad de los nacionalismos». Inguruak: Revista Vasca de Sociología y Ciencia Política, 27, septiembre, 75-93.

- (2002). «Sobre el papel de la política en la comprensión modernista del origen de las naciones: Ernest Gellner frente a Paul Brass». Papers: Revista de Sociologia, 67, 149-176.

GELLNER, Ernest (1983). Naciones y nacionalismo. Madrid: Alianza Universidad, 1988.

Greenfeld, Liah (1999). Nacionalisme i modernitat. Catarroja-Valencia: Afers. Universitat de València.

GuibernaU, Montserrat (1996). Los nacionalismos. Barcelona: Ariel.

HobSBAwM, E.J. (1992). Naciones y nacionalismo desde 1780. Barcelona: Crítica.

Kedourie, Elie (1966). Nationalism. Londres: Hutchinson University Library.

KyMlickA, Will (2001). Politics in the vernacular: Nationalism, multiculturalism and citizenship. Oxford: Oxford University Press.

LAEGAARD, Sune (2007). "Liberal nationalism and the nationalisation of liberal values». Nations and Nationalism, 13 (1), 37-55.

LAWRENCE, Paul (2005). Nationalism: History and theory. Harlow (Inglaterra): Pearson.

Llobera, Josep R. (1994). The God of Modernity. The Development of Nationalism in Western Europe. Oxford: Berg Publishers.

- (1999). "Recent theories of nationalism». Barcelona: Institut de Ciències Polítiques i Socials. Working Paper, 164. 
MAIZ SuÁREZ, Ramón (1997). «Retos contemporáneos de la política (II): Los nacionalismos». En: ÁGUILA, R. del. Manual de ciencia política. Valladolid: Trotta, capítulo $19,477-505$.

- (2004). «Per Modum Unius: Más allá de la dicotomía nacionalismo cívico/nacionalismo étnico». En: GurrutXaga, A. El Presente del Estado-nación. Bilbao: UPV, 107-130.

MarX, Karl; Engels, Federich (1971). Manifiesto del Partido Comunista. Pekín: Ediciones en Lenguas Extranjeras.

ÖZKIRIMLI, Umut (2000). Theories of nationalism. A critical introduction. Houndmills: Palgrave.

RodríGUEZ ABASCAL, Luis (2000). Las fronteras del nacionalismo. Madrid: Centro de Estudios Políticos y Constitucionales.

SMITH, Anthony D. (1986). The ethnic origins of nations. Oxford: Blackwell.

- (1998). Nationalism and modernism. Londres: Routledge.

- (2001). Nationalism. Cambridge: Polity.

- (2003). Chosen peoples. Sacred sources of national identity. Oxford: Oxford University Press.

TAiBO, Carlos (dir.) (2007). Nacionalismo español. Esencias, memoria e instituciones. Madrid: Catarata.

TAMIR, Yael (1993). Liberal nationalism. Princeton: Princeton University Press.

Tratado de la Unión Europea (1992), conocido como Tratado de Maastricht. Consultado en

[http://eur-lex.europa.eu/es/treaties/dat/11992M/htm/11992M.html]

VisvanATHan, Shiv (2006). «Nation». Theory, Culture \& Society, vol. 23 (2-3), 533-538.

WEBER, Max (2002). La ética protestante y el «espíritu» del capitalismo. Madrid: Alianza.

- (2007). Sociología del poder: Los tipos de dominación. Madrid: Alianza. 